.5

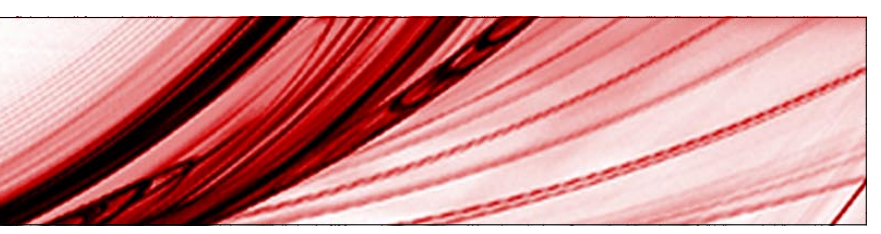

\title{
Characterization of complexities in combustion instability in a lean premixed gas-turbine model combustor
}

Hiroshi Gotoda, Masahito Amano, Takaya Miyano, Takuya Ikawa, Koshiro Maki et al.

Citation: Chaos 22, 043128 (2012); doi: 10.1063/1.4766589

View online: http://dx.doi.org/10.1063/1.4766589

View Table of Contents: http://chaos.aip.org/resource/1/CHAOEH/v22/i4

Published by the American Institute of Physics.

\section{Related Articles}

Reduced hierarchy equations of motion approach with Drude plus Brownian spectral distribution: Probing electron transfer processes by means of two-dimensional correlation spectroscopy

J. Chem. Phys. 137, 22A550 (2012)

Rectified Brownian transport in corrugated channels: Fractional Brownian motion and Lévy flights

J. Chem. Phys. 137, 174101 (2012)

Brownian dynamics simulations with hard-body interactions: Spherical particles

J. Chem. Phys. 137, 164108 (2012)

Noncolliding Brownian motion with drift and time-dependent Stieltjes-Wigert determinantal point process J. Math. Phys. 53, 103305 (2012)

Non-Markovian reduced dynamics based upon a hierarchical effective-mode representation

J. Chem. Phys. 137, 144107 (2012)

\section{Additional information on Chaos}

Journal Homepage: http://chaos.aip.org/

Journal Information: http://chaos.aip.org/about/about_the_journal

Top downloads: http://chaos.aip.org/features/most_downloaded

Information for Authors: http://chaos.aip.org/authors

\section{ADVERTISEMENT}

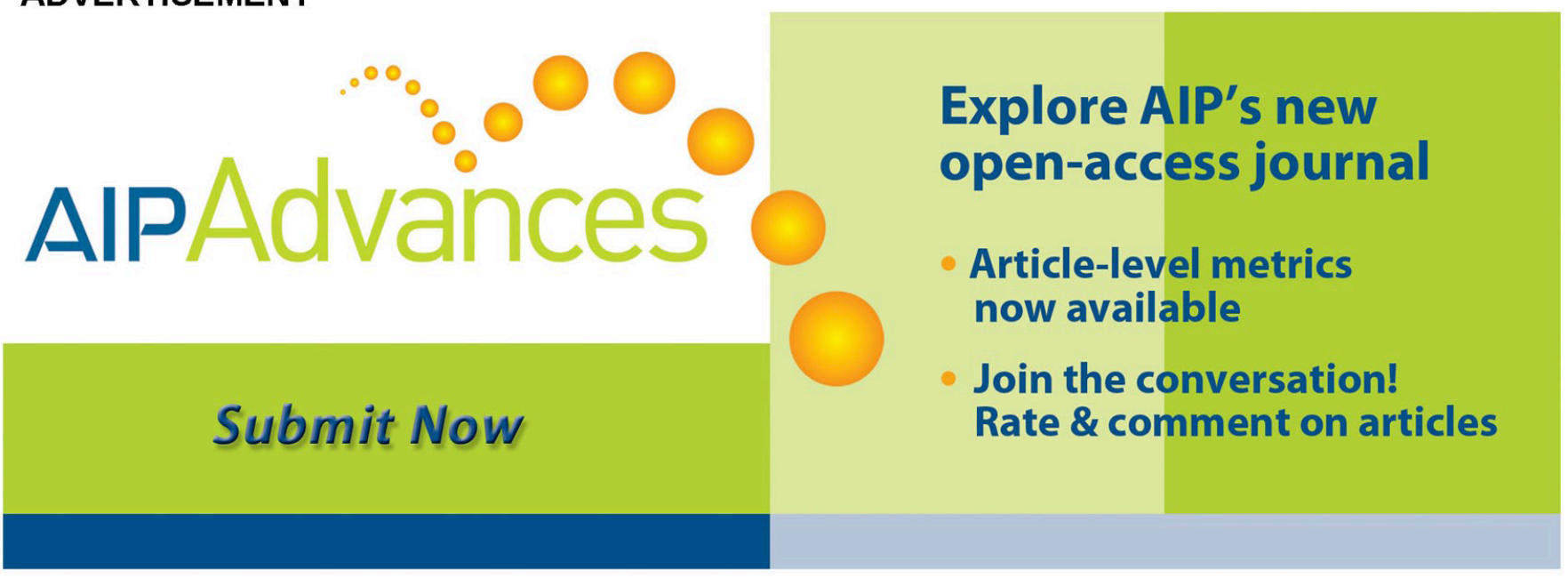




\title{
Characterization of complexities in combustion instability in a lean premixed gas-turbine model combustor
}

\author{
Hiroshi Gotoda, ${ }^{1, a)}$ Masahito Amano, ${ }^{1}$ Takaya Miyano, ${ }^{1, b)}$ Takuya lkawa, ${ }^{1}$ Koshiro Maki, ${ }^{1}$ \\ and Shigeru Tachibana ${ }^{2, c)}$ \\ ${ }^{1}$ Department of Mechanical Engineering, Ritsumeikan University, 1-1-1 Nojihigashi, Kusatsu-shi, \\ Shiga 525-8577, Japan \\ ${ }^{2}$ Aerospace Research and Development Directorate, Japan Aerospace Exploration Agency, \\ 7-44-1 Jindaiji-Higashii, Chofu, Tokyo 182-8522, Japan
}

(Received 28 March 2012; accepted 10 October 2012; published online 30 November 2012)

An understanding of the physical process underlying combustion instability leading to lean blowout is of current interest in modern combustion physics as well as in nonlinear science. In this study, the characterization of complexities in combustion instability in a lean premixed gas-turbine combustor, which is of fundamental and practical importance for combustion systems, has been carried out from the viewpoint of nonlinear dynamics, focusing on characterizing the dynamic behavior of combustion instability close to lean blowout. The use of nonlinear time series analysis involving permutation entropy in combination with a surrogate data method, multifractal analysis, and nonlinear forecasting based on a radial basis function network allows us to capture the signature of self-affine structures and chaotic oscillations in combustion instability. Our results have not been reported in previous papers on combustion phenomena, in particular, thermoacoustic combustion oscillations.

\section{INTRODUCTION}

Lean premixed combustion is becoming an effective way for reducing nitrogen oxide $\left(\mathrm{NO}_{\mathrm{x}}\right)$ emission from gasturbine engines. However, it is more susceptible to the excitation of combustion instabilities such as lean blowout, thermoacoustic combustion oscillations, and flame flashback, which is a major issue that limits the development of propulsion and land-based gas-turbine engines. Thermoacoustic combustion oscillations, caused by closed-loop coupling between unsteady pressure and heat-release fluctuations referred to as the Rayleigh criterion, lead to serious damage in combustors and are detrimental to the operation of engines. A complex interaction between the combustion process and acoustic field inside the combustors generates the

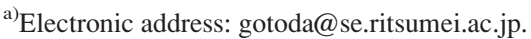

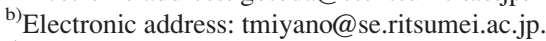

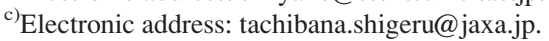

nonlinear dynamical behavior of thermoacoustic combustion oscillations. Details of the physical mechanism of the excitation of thermoacoustic combustion oscillations in various types of laboratory-scale gas-turbine combustor have been discussed in a recent review. ${ }^{1}$ In most studies, ${ }^{1-9}$ a linear analysis such as power spectrum analysis has been applied to the pressure and heat-release fluctuations with the aim of characterizing the dynamics underlying thermoacoustic combustion oscillations. This method is one of the common and conventional methods of characterizing unsteady combustion modes of an observational time series, but may be insufficient for revealing the nonlinear nature of combustion instability. In fact, combustion instability is roughly classified by the power spectrum into stable combustion represented by a limit cycle with a small oscillation amplitude and unstable combustion represented by a limit cycle with a large oscillation amplitude and well-defined oscillation frequencies. ${ }^{1}$ This indicates that the power spectrum may have limited use in characterizing the complex dynamics underlying combustion instability.

The nonlinear time series approach inspired by chaos theory conveys useful information for clarifying the nonlinear properties of complex dynamics, and its utilization has led to the possibility of uncovering information about the underlying dynamics of flame or combustion instability. In recent years, it has been applied to the temporal behavior of the unstable combustion state observed not only in experiments (e.g., a thermal pulse combustor, ${ }^{10}$ a ducted premixed combustor, $^{11-13}$ a spark ignition engine, ${ }^{14,15}$ and swirling premixed flames ${ }^{16}$ ) but also in the numerical solutions of sets of nonlinear differential equations derived from first principles (e.g., a thermal pulse combustor, ${ }^{17}$ detonation, ${ }^{18,19}$ and a spark ignition engine ${ }^{20}$ ). In previous studies ${ }^{6,11-13}$ relevant to thermoacoustic combustion oscillations, the Grassberger-Procaccia (GP) algorithm, ${ }^{21}$ used to obtain the correlation dimension as a class of the fractal dimension from an observational time series, was used to characterize the nonlinear nature. In particular, the usefulness of the GP 
algorithm in characterizing the nonlinear nature of pressure fluctuations in thermoacoustic combustion oscillations was emphasized by Kabiraj and co-workers. ${ }^{12}$ However, the GP algorithm often leads to a misdiagnosis in distinguishing chaos from temporally correlated stochastic processes or in testing for low-dimensional chaos from a time series. ${ }^{22}$ Diks, ${ }^{23}$ Yu et al., ${ }^{24}$ and Small et al. ${ }^{25}$ have recently proposed improved versions of the GP algorithm. Without using the correlation dimension and instead by estimating the degree of parallelism of neighboring trajectories in the phase space reconstructed from a time series, we have recently reported the possibility that pressure fluctuations at an equivalence ratio of $\phi=0.43$ near lean blowout (see Fig. 1) in a lean premixed gas-turbine model combustor are ascribed to stochastic processes, whereas those at $\phi=0.45$ are ascribed to chaotic oscillations with slow amplitude modulation. ${ }^{25}$ The importance of the degree of parallelism has been shown in a previous study regarding flame front instability induced by buoyancy/swirl coupling. ${ }^{16}$

As mentioned above, the relevance of chaos to thermoacoustic combustion oscillations has been shown by nonlinear time series analysis, even in the above previous work. ${ }^{26}$ To obtain better understanding of the nonlinear dynamics in combustion instability, the characterization of complexities of combustion instability close to lean blowout is needed from different viewpoints, which will be of significance in not only combustion science and physics but also nonlinear dynamics. Our purpose in this study is to conduct a more indepth investigation of the complexities of combustion instability in a lean premixed gas-turbine model combustor from the viewpoints of permutation entropy, multifractal structure, and nonlinear forecasting based on a neural network. We first use the mathematical method devised by Bandt and Pompe ${ }^{27}$ to estimate permutation entropy. Permutation entropy represents the degree of randomness estimated from a sequence of ranks in the values of a time series, as a coarse-grained duplication of the time series. This method was used to characterize the dynamic properties of a different type of flame front instability. ${ }^{28}$ Another simple and useful time series analysis for distinguishing chaos from stochastic processes, referred

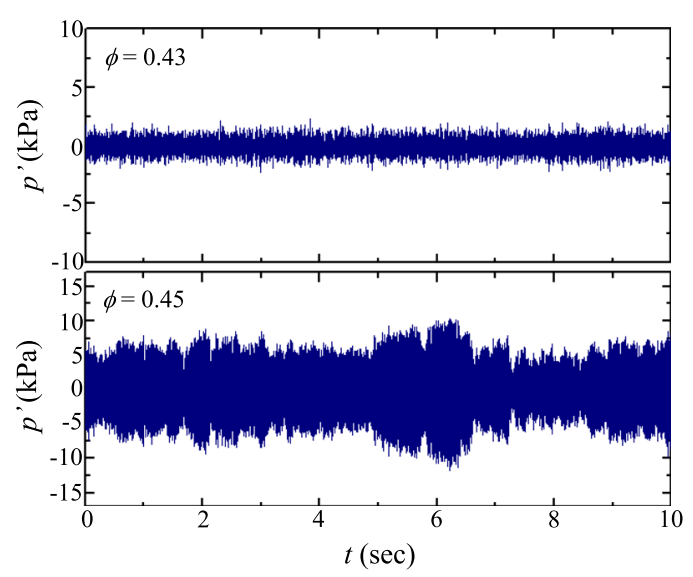

FIG. 1. Time variations in pressure fluctuation $p^{\prime}$ at equivalence ratios $\phi=0.43$ and 0.45 . The upper figure (lower figure) shows the time variation in $p^{\prime}$ for combustion instability near lean blowout (for combustion oscillation with a small modulation in amplitude). to as the $0-1$ test, has been introduced by Gottwald and Melbourne. $^{29}$ The 0-1 test classifies a given time series into one of two opposite classes: deterministic behavior and uncorrelated random behavior. However, in this study, we estimate the degree of visible determinism in the dynamic behavior, rather than make a judgment of whether the dynamical behavior is deterministic or stochastic. For this reason, we do not apply the 0-1 test in this study. Instead, the amplitude-adjusted Fourier transform (AAFT) surrogate method $^{30}$ and the cycle surrogate method ${ }^{31,32}$ are applied to evaluate the statistical significance of the estimates for the permutation entropy. Second, we use multifractal analysis to examine the fractal structure in the phase space constructed from the time series data of pressure fluctuations. This allows the degree of self-similarity to be evaluated as a spectrum, and has recently been applied to study the dynamic behavior of a spark ignition engine. ${ }^{15,20}$ We do not use improved versions of the GP algorithm ${ }^{23-25}$ because they cannot assess multifractality. The dynamic behavior of deterministic chaos has the important features of short-term predictability and long-term unpredictability. In nonlinear physics, nonlinear forecasting based on orbital instability in the phase space or neural network theory has been shown to ensure good performance in the short-term prediction of deterministic chaos. ${ }^{26,33-50}$ We use a generalized radial basis function (GRBF) network, ${ }^{33,38,39,46}$ which is based on regularization theory. ${ }^{40}$ This can be considered to be an inverse approach in the sense that the underlying dynamics is expressed by a predictive model constructed from the observed temporal behavior. The importance of the inverse approach has been discussed in studies of crystal growth ${ }^{38}$ and a blast furnace. ${ }^{46}$

This paper is organized as follows. In Sec. II, we briefly describe the experimental system and methods used in this work. In Sec. III, we present the central ideas behind the mathematics of time series analysis. We present the results and discussion in Sec. IV and our conclusions in Sec. V.

\section{EXPERIMENTAL SYSTEM AND METHODS}

The experimental system we used in this study is identical to that used in previous studies. ${ }^{8,9,26}$ It is mainly composed of a blower, an electric heater, an axial swirler, a combustion chamber with a length of $630 \mathrm{~mm}$ and a $100 \times 100 \mathrm{~mm}^{2}$ cross section, and a water-cooled stainlesssteel duct (see Fig. 1 of Ref. 26). The inlet air preheated at $700 \mathrm{~K}$ is delivered to the combustion chamber at a mass flow rate of $78 \mathrm{~g} / \mathrm{s}$. Methane gas is used as the main fuel, which is injected through multiple orifices $260 \mathrm{~mm}$ upstream of the inlet of the combustion chamber. The swirler with a vane angle of $45^{\circ}$ relative to the inlet premixture stream is used as a flame holder in this study. As in previous works, ${ }^{8,9}$ active control by secondary fuel injection is not applied in this study so that we can focus on characterizing the complexities of combustion instability. The equivalence ratio of the methane/air premixture $\phi$ is varied from 0.43 to 0.47 because, under these conditions, the dynamic behavior of combustion instability near lean blowout undergoes a transition to chaotic oscillations. The pressure fluctuations of combustion instability $p^{\prime}$ are obtained using a pressure transducer 
(Kulite Semiconductor Products, Model XTEL-190-15G) placed on the wall of the combustion chamber with the aim of investigating the dynamic behavior of combustion instability. ${ }^{26}$ The pressure transducer is set $10 \mathrm{~mm}$ downstream of the inlet of the combustion chamber because the effect of thermoacoustic coupling strongly appears in the time series of pressure fluctuations. Nonlinear time series analysis is applied to the time series data of $p^{\prime}$ at a sampling frequency of $5.12 \mathrm{kHz}$.

\section{MATHEMATICAL TREATMENT USED FOR NONLINEAR TIME SERIES ANALYSIS}

\section{A. Permutation entropy in combination with surrogate data method}

Kolmogorov-Sinai (KS) entropy is an invariant measure of the complexity of dynamics. Bandt and Pompe ${ }^{27}$ have recently introduced permutation entropy as a coarse-grained version of the KS entropy that can be estimated by a convenient algorithm using a time series. Given a sequence with embedding dimension $D$, we index all possible permutations ( $D$ ! permutations) of order $D \geq 2$ as $\pi$. Each permutation represents a coarse-grained pattern of the dynamic behavior when the sequence consists of $D$ successive data points taken from a time series. We first count the number of permutations denoted as $q(\pi)$ for all vectors $\mathbf{X}(t)=\left(p^{\prime}(t)\right.$, $\left.p^{\prime}(t+1), \ldots, p^{\prime}(t+D-1)\right)$ consisting of sequences of order $D$. We then calculate the relative frequency for each permutation to obtain $p(\pi)=q(\pi) /(N-D+1)$. The permutation entropy is defined as

$$
H(D)=-\sum_{\pi} p(\pi) \log _{2} p(\pi) .
$$

The permutation entropy can be normalized as follows with respect to the maximum permutation entropy, which is obtained for completely random processes:

$$
\bar{h}=\frac{H(D)}{\log _{2} D !},
$$

where $0 \leq \bar{h} \leq 1$. The lower bound corresponds to a monotonically increasing or decreasing process, while the upper bound corresponds to a completely random process.

The surrogate method first introduced by Theiler $\mathrm{et} \mathrm{al.} .^{30}$ is a type of statistical test used in combination with nonlinear time series analysis to test for weak evidence of deterministic chaos. In this method, we first postulate a null hypothesis about the dynamics generating some statistical properties of a given time series. For instance, under the null hypothesis that irregular components of pressure fluctuations represent a stochastic process, we generate sets of surrogate time series such that they possess the same power spectra as those of the original time series. This can be conducted using the AAFT surrogate method. The surrogate time series entirely preserve the power spectra owing to the regular oscillations included in the original time series; however, they lose the determinism (chaos) generating the irregular components, if any, of the original time series. For periodic cycles with slow amplitude modulation included in pressure fluctuations, ${ }^{26}$ the surrogate method based on shuffling individual cycles within a time series, referred to as the cycle surrogate data, is more useful for testing for determinism in the case of slow amplitude modulation. Referring to previous studies, ${ }^{31,32}$ under the null hypothesis that each cycle is independent of its adjacent cycle and that no determinism is visible in pitch fluctuations of the time series, we generate a ten-cycle surrogate time series.

The next step in the surrogate method is to estimate appropriate statistics such as permutation entropy for both the original time series and the surrogate series. Then, we calculate the mean and variance of the estimated statistics for the surrogate data and conduct a $t$-statistical test to evaluate the statistical significance of the difference in the estimated statistics between the original and surrogate data. When the $t$-test statistic exceeds the critical $t$-value for the given degrees of freedom, the null hypothesis is rejected. This rejection implies that the irregular components of the original time series stem from a deterministic process, which can be interpreted as possible evidence of the existence of chaos.

\section{B. Multifractal analysis}

A multifractal structure exhibits various self-similarities in the phase space constructed from time series data, which is generally characterized by a singularity spectrum. The singularity spectrum represents the fractal dimension of interwoven sets in the attractor. It provides us with an intuitive understanding of the multifractal structure in terms of the singularity strength and has been applied in recent combustion research. ${ }^{15,20}$ Note that in previous studies, ${ }^{15,20}$ the singularity spectrum was directly estimated for positive time series data.

On the basis of Takens' embedding theorem, ${ }^{51}$ the phase space is constructed from the time series data of the pressure fluctuations $p^{\prime}$. The time-delayed coordinates used for the construction of the phase space are expressed as

$\mathbf{x}\left(t_{i}\right)=\left(p^{\prime}\left(t_{i}\right), p^{\prime}\left(t_{i}+\tau\right), p^{\prime}\left(t_{i}+2 \tau\right), \ldots, p^{\prime}\left(t_{i}+(D+1) \tau\right)\right)$,

where $i=0,1, \ldots, n$ ( $n$ is the data number of the time series), $\mathbf{x}\left(t_{i}\right)$ are the phase space vectors, $p^{\prime}\left(t_{i}\right)$ are the pressure fluctuations at time $t_{i}, D$ is the embedding dimension, that is, the dimension of the phase space, and $\tau$ is the time lag. The value of $D$ used to obtain the singular spectrum is set to 3 in this work with reference to a previous study. ${ }^{52}$ If the time lag is too small, then the elements of the phase space vectors are strongly correlated. If the time lag is too large, then the correlation is lost completely. An appropriate choice for the time lag is made using mutual information. ${ }^{26}$

In this work, we directly estimate the singularity spectrum using the standard box-counting method. The probability measure in the $i$ th box, which is divided into boxes of size $\varepsilon$ in the support of the phase space, is scaled with the singularity strength as

$$
p_{i}(\varepsilon)=\varepsilon^{\alpha_{i}},
$$


where $p_{i}$ is the probability measure in the $i$ th box and $\alpha_{i}$ is the singularity strength characterizing the scaling in the $i$ th box. The number of boxes $N(\alpha)$ for which the probability has singularity strength between $\alpha$ and $d \alpha$ scales with $f(\alpha)$ according to the power law of $p_{i}(\varepsilon)$ as follows:

$$
N(\alpha)=\varepsilon^{-f(\alpha)} .
$$

The partition function is obtained for the parameter $q$, which can vary from $-\infty$ to $+\infty$,

$$
Z(q)=\sum_{i} p_{i}(\varepsilon)^{q}
$$

A scaling exponent function $\tau(q)$ can be estimated from the slope of the linear part of the $\ln Z(q)$ versus $\ln \varepsilon$ curve for different $q$ using

$$
\tau(q)=\lim _{\varepsilon \rightarrow 0} \frac{\ln Z(q)}{\ln \frac{1}{\varepsilon}} .
$$

The singularity strength $\alpha$ and its spectrum $f(\alpha)$ are finally obtained from the following equations obtained by the Legendre transformation:

$$
\begin{gathered}
\alpha=\frac{d \tau(q)}{d q}, \\
f(\alpha)=q \alpha-\tau(q) .
\end{gathered}
$$

The generalized dimension $D_{q}$ is expressed as follows:

$$
D_{q}=\frac{\tau(q)}{q-1}
$$

Note that $D_{q}$ at $q=0$ corresponds to the capacity dimension in the phase space. A schematic of the singularity spectrum $f(\alpha)$ as a function of singularity strength $\alpha$ is shown in Fig. 2. When $q=0$, the value of $f(\alpha)$ becomes maximum. From Eqs. (9) and (10), the maximum value of the singularity spectrum $f(\alpha)_{\max }$ corresponds to the capacity dimension. Therefore, $f(\alpha)_{\max }$ is estimated for different equivalence ratios in this work. In addition, the degree of multifractality $\Delta \alpha\left(=\alpha_{\max }-\alpha_{\min }\right)$ is also estimated. As will be shown in

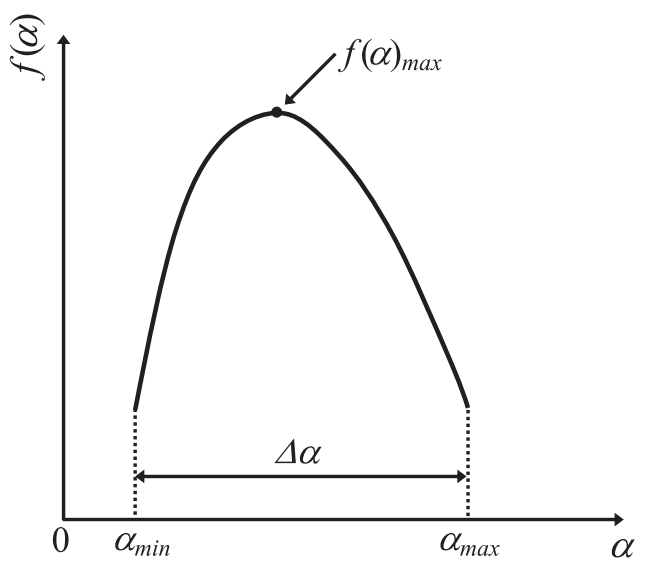

FIG. 2. Schematic of singularity spectrum.
Fig. 5, the singularity spectrum does not intersect at an axis of the singularity strength, but we estimate the multifractality $\Delta \alpha$ in this work (see Fig. 2) similarly to in a previous study ${ }^{20}$ (note that a clear definition of multifractality was not given in the previous study ${ }^{20}$ ). In a preliminary test, the value of $f(\alpha)_{\max }$ calculated for the time series data of the solution of the Lorenz equations is nearly in accord with the capacity dimension $(=2.07)$, and the validity of the calculation code applied in this work is confirmed.

\section{Nonlinear forecasting based on neural network}

We utilize a GRBF neural network as a nonlinear forecasting method to capture the predictability of the dynamic behavior of combustion instability close to lean blowout. Previous applications of GRBF networks to complex time series have been reported. ${ }^{38,46}$ We refer to $\mathbf{p}^{\prime}\left(t_{i}\right)$ as the input vectors consisting of lagged sequences of data points of pressure fluctuations and $p^{\prime}\left(t_{i}+T \Delta t\right)$ as the corresponding data point $T$ time steps in the future. Predictions of $\hat{p}^{\prime}\left(t_{i}+T \Delta t\right)$ about the corresponding actual value $p^{\prime}\left(t_{i}+T \Delta t\right)$ are made using the following input-output mapping, i.e., a network of radial basis functions:

$$
\hat{p}^{\prime}\left(t_{i}+T \Delta t\right)=\sum_{h=1}^{N_{h}} c_{h} \exp \left\{-\beta_{h}\left\|\mathbf{p}^{\prime}\left(t_{i}\right)-\gamma_{h}\right\|^{2}\right\},
$$

where $\mathbf{p}^{\prime}\left(t_{i}\right)=\left(p^{\prime}\left(t_{i}\right), p^{\prime}\left(t_{i}-\tau\right), p^{\prime}\left(t_{i}-2 \tau\right), \ldots, p^{\prime}\left(t_{i}-(D+1) \tau\right)\right)$, $N_{h}$ is the number of Gaussian functions, i.e., the basis functions, $c_{h}$ are the weight coefficients, and $\beta_{h}$ and $\gamma_{h}$ are the scalar and vector parameters determining the shapes of the basis functions, respectively. In GRBF networks, $\beta_{h}$ and $\gamma_{h}$ are optimized by applying a learning algorithm such as stochastic gradient descent to the time series. The GRBF network approximates the dynamics underlying the time series using a single model defined by Eq. (11) over the entire phase space. In this sense, the GRBF network is a class of global predictive methods.

In this study, we optimize the parameters $c_{h}, \beta_{h}$, and $\gamma_{h}$ using stochastic gradient descent such that the parameters minimize the approximation error $E$ between $\hat{p}^{\prime}\left(t_{i}+T \Delta t\right)$ and $p^{\prime}\left(t_{i}+T \Delta t\right)$, defined by

$$
E=\frac{1}{N} \sum_{i=1}^{N}\left[p^{\prime}\left(t_{i}+T \Delta t\right)-\hat{p}^{\prime}\left(t_{i}+T \Delta t\right)\right]^{2} .
$$

Stochastic gradient descent involves the following set of ordinary differential equations:

$$
\begin{gathered}
\frac{d c_{h}}{d s}=\omega_{1} \frac{\partial E}{\partial c_{h}}, \\
\frac{d \beta_{h}}{d s}=\omega_{2} \frac{\partial E}{\partial \beta_{h}}+\eta(s), \\
\frac{d \gamma_{h}}{d s}=\omega_{3} \frac{\partial E}{\partial \gamma_{h}}+\eta(s),
\end{gathered}
$$

where $s$ is a time parameter in the learning process, $\omega_{1}, \omega_{2}$, and $\omega_{3}>0$ are appropriately chosen learning rates, and $\eta(s)$ 
is a Gaussian white noise that has a mean of 0 and an appropriate variance and is added to the right-hand side of Eqs. (14) and (15) every appropriately chosen time interval to allow the learning system to escape from local minima with large approximation errors. We keep applying Eqs. (13)-(15) until $E$ is reduced to a sufficiently small value. The initial $c_{h}, \beta_{h}$, and $\gamma_{h}$ are set to random numbers lying between -1 and 1 . In a preliminary test, the validity of the algorithm used in this work is confirmed by predicting the time series data of the low-dimensional deterministic chaos produced using the Henon map and Lorentz equations.

\section{RESULTS AND DISCUSSION}

The normalized permutation entropy $\bar{h}$ as a function of the embedding dimension $D$ is shown in Fig. 3 for the original time series, AAFT surrogate time series, and cycle surrogate time series data of the pressure oscillations at an equivalence ratio of $\phi=0.45$. The physical mechanism of the onset of combustion oscillations with slow amplitude modulation was phenomenologically explained in previous studies. ${ }^{8,9,26}$ As shown in Fig. $3, \bar{h}$ for the original time series data gradually decreases with increasing $D$. This trend basically corresponds to that obtained in previous studies of acoustic emission by an anomalous discharge in a plasma system $^{53}$ and flame front instability induced by radiative heat loss. ${ }^{28}$ A similar trend for $\bar{h}$ with respect to $D$ is observed for both AAFT and cycle surrogate time series data. However, the estimates of $\bar{h}$ for the original time series become considerably lower than those for the surrogate series as $D$ increases. The null hypotheses can be rejected by a twosided $t$-test for $\bar{h}$ at $5 \%$ reliability. Our previous study using nonlinear time series analysis ${ }^{26}$ suggested that pressure fluc-

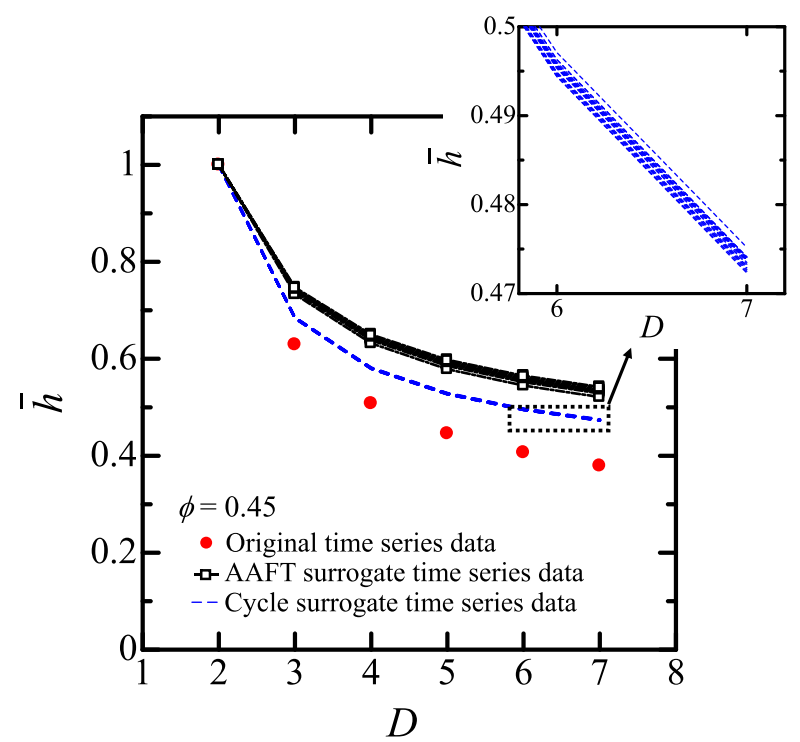

FIG. 3. Variation in normalized permutation entropy $\bar{h}$ as a function of embedding dimension $D$ : original data $(\bullet)$, AAFT surrogate data $(\square$ and lines) and cycle surrogate data (lines) at equivalence ratio $\phi=0.45$. The temperature of preheated inlet air is $700 \mathrm{~K}$. The mass flow rate of preheated inlet air is $78 \mathrm{~g} / \mathrm{s}$. The difference between the values of $\bar{h}$ estimated for the original and both surrogate time series data is significantly large, which shows that the null hypotheses can be rejected.

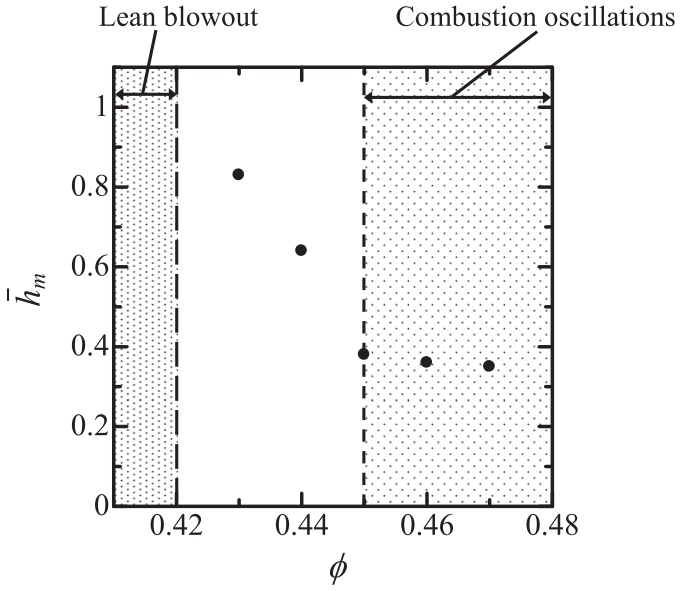

FIG. 4. Variation in minimum of normalized permutation entropy $\bar{h}_{m}$ as a function of equivalence ratio $\phi$. The experimental conditions of preheated inlet air flow are the same as those in Fig. 3.

tuations at $\phi=0.46$ represent chaotic oscillations. On this basis, the result in Fig. 3 suggests that pressure fluctuations at $\phi=0.45$ are due to chaotic oscillations. The minimum normalized permutation entropy $\bar{h}_{m}$ for the original time series data is shown in Fig. 4 as a function of the equivalence ratio $\phi$. Here, note that the minimum $\bar{h}_{m}$ for a change in the embedding dimension $D$ is plotted in Fig. 4. $\bar{h}_{m}$ at $\phi=0.43$ is approximately 0.84 , indicating that the dynamic behavior near lean blowout is a stochastic process. $\bar{h}_{m}$ decreases significantly as $\phi$ increases, which means that the degree of complexity in the dynamic behavior of pressure fluctuations decreases. When combustion oscillations with slow amplitude modulation begin to appear, the degree of complexity in the dynamic behavior becomes nearly constant.

As mentioned in Sec. I, the correlation dimension method, which is a conventional method of quantifying the degree of self-similarity in the phase space, has been widely applied in studies of thermoacoustic combustion oscillations, ${ }^{6,11-13}$ however, information on self-similarity in combustion dynamics is limited in the sense that a structure with self-similarity is expressed as only a single fractal dimension. In contrast, multifractal analysis, which allows the degree of self-similarity to be evaluated as a spectrum, is useful for quantifying details of the fractal structure and has recently been used to study the dynamic behavior of a spark ignition engine. ${ }^{15,20}$ As in previous studies, ${ }^{15,20}$ we conduct a multifractal analysis of a three-dimensional phase space constructed from time variations in pressure fluctuations. Note that the previous studies ${ }^{15,20}$ directly performed a multifractal analysis of the time variation in pressure fluctuations of a spark ignition engine, that is, a one-dimensional structure, without considering Takens' embedding theorem. ${ }^{51}$ In this sense, compared with the analysis in the previous studies, ${ }^{15,20}$ the multifractal analysis carried out in this work is extended to reveal a structure with self-similarity in the phase space. A similar analysis has been applied to a lowdimensional phase space constructed from the time variations in the numerical solutions obtained for various types of nonlinear dynamical system, such as the Henon map and logistic map. ${ }^{52}$ The singularity spectrum $f(\alpha)$ as a function 


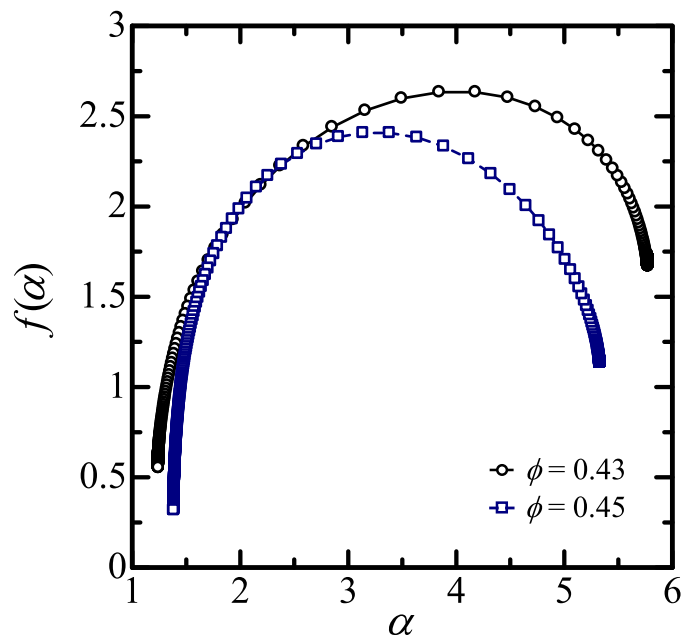

FIG. 5. Singularity spectra $f(\alpha)$ as functions of singularity strength $\alpha$ at equivalence ratios $\phi=0.43$ and 0.45 . The experimental conditions of heated inlet air flow are the same as those in Fig. 3. The shapes of $f(\alpha)$ for $\phi=0.43$ and 0.45 are unimodal and downward concave, which indicates the existence of a multifractal structure.

of singularity strength $\alpha$ at $\phi=0.43$ and 0.45 is shown in Fig. 5. The shape of $f(\alpha)$ in both cases is clearly unimodal and downward concave. This indicates that both the dynamics in combustion instability near lean blowout and the combustion oscillations with slow amplitude modulation have a multifractal structure. An interesting result to note in Fig. 5 is that the maximum $f(\alpha)$ at $\phi=0.43$ is a noninteger $(=2.7)$ smaller than the embedding dimension $(D=3)$. In our previous work, ${ }^{26}$ we concluded that the dynamic behavior of pressure fluctuations near lean blowout is stochastic. On the basis of the result obtained in Fig. 5, it is conceivable that the dynamic behavior of combustion instability near lean blowout may exhibit a self-affine structure and fractional Brownian motion. This indicates that the important nonlinear nature of pressure fluctuations near lean blowout, which cannot be discussed by measuring the parallelism of neighboring trajectories in the phase space, ${ }^{16,26}$ can be extracted by multi-

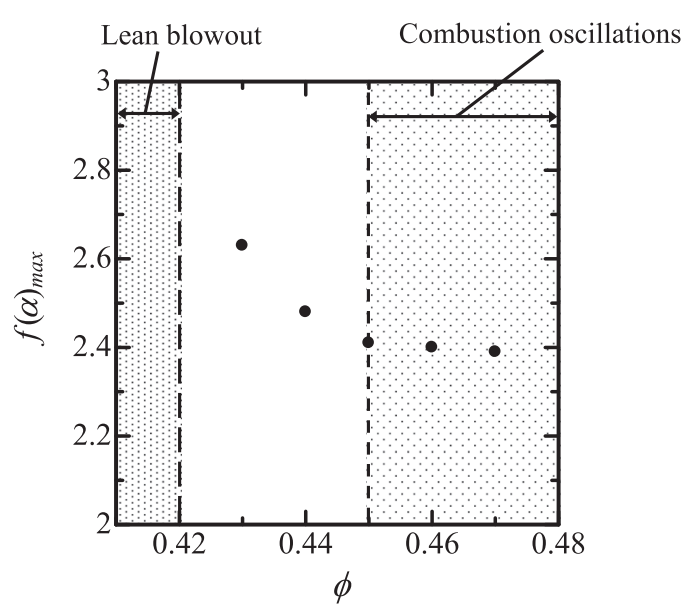

FIG. 6. Variation in maximum singularity spectrum $f(\alpha)_{\max }$ as a function of equivalence ratio $\phi$. The experimental conditions of preheated inlet air flow are the same as those in Fig. 3. The capacity dimension reaches 2.4 with the onset of combustion oscillations with slow amplitude modulation, indicating chaotic oscillations. fractal analysis. The maximum singularity spectrum $f(\alpha)_{\max }$, which corresponds to the capacity dimension, is shown in Fig. 6 as a function of $\phi$. With increasing $\phi, f(\alpha)_{\max }$ gradually decreases, reaching 2.4 when combustion oscillations with slow amplitude modulation appear. Generally, the Lorenz model is not appropriate as a platform for considering combustion instability in real systems because it is an oversimplified physical model derived from the Boussinesq equations under a free-boundary condition to purify the dynamic behavior in unstable phenomena generated by buoyancydriven hydrodynamic instabilities. Nevertheless, the Lorenz model is useful for the purpose of our study because it enables us to discuss the degree of complexity of dynamic instability. ${ }^{26}$ The translation error for combustion oscillations with slow amplitude modulation is close to that for the lowdimensional deterministic chaos produced using the Lorenz equations. $^{26}$ Although $f(\alpha)_{\max }$ for combustion oscillations with slow amplitude modulation is larger than that for the Lorenz equations, on the basis of the result obtained using the translation error ${ }^{26}$ and multifractal analysis, it is concluded that the dynamic behavior of combustion oscillations with slow amplitude modulation is low-dimensional chaos. The multifractality $\Delta \alpha$ as a function of $\phi$ is shown in Fig. 7. $\Delta \alpha$ gradually decreases with increasing $\phi$, which means that the multifractality of the dynamic behavior of pressure fluctuations decreases as combustion oscillations with slow amplitude modulation occur. That is, the multifractality of combustion instability near lean blowout becomes stronger. As mentioned in Sec. I, the correlation dimension of trajectories in the phase space obtained by the GP method ${ }^{21}$ has recently been used more frequently to quantify the fractal structure of thermoacoustic combustion oscillations in a ducted premixed combustor. ${ }^{11-13}$ It has also been used to characterize the dynamic behavior of flame front instability. ${ }^{54,55}$ However, the results obtained in this work suggest that multifractal analysis is a more suitable analytical method of extracting details of fractal structures than the correlation dimension method of estimating a single fractal

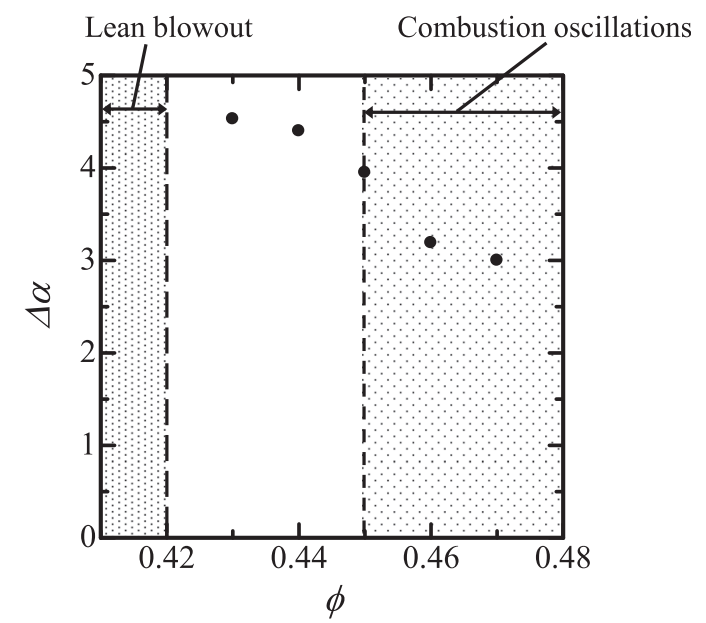

FIG. 7. Variation in multifractality $\Delta \alpha$ as a function of equivalence ratio $\phi$. The experimental conditions of preheated inlet air flow are the same as those in Fig. 3. The multifractality of the dynamic behavior of pressure fluctuations decreases with the onset of combustion oscillations with slow amplitude modulation. 
dimension and is of great importance for the combustion community.

A well-known feature of fractional Brownian motion is that the degree of inconsistency between predicted and actual dynamic behaviors significantly increases with time. In other words, evidence of fractional Brownian motion is provided by one-step-ahead and multistep-ahead predictions of successive pressure fluctuations. If the following scaling law is satisfied, the dynamic behavior of pressure fluctuations can be said to exhibit fractional Brownian motion with the scaling exponent $H(0<H<1)$. Note that $H$ corresponds to the Hurst exponent

$$
\frac{E_{p}(T)}{E_{p}(1)}=T^{H}
$$

Here, $E_{p}$ is the prediction error and $T$ is the prediction step.

Equation (16) can be rewritten as

$$
\ln \frac{E_{p}(T)}{E_{p}(1)}=H \ln T
$$

$H$ can be estimated as the slope of the $\ln \left[E_{p}(T) / E_{p}(1)\right]-$ $\ln T$ plot. The algorithm based on the scaling properties of fractional Brownian motion was first introduced by Tsonis and Elsner. ${ }^{36}$ It has been used in previous studies of crystal growth $^{38}$ and a blast furnace. ${ }^{46}$ As was shown in the result of multifractal analysis, the dynamic behavior of combustion instability near lean blowout $(\phi=0.43)$ may exhibit fractional Brownian motion. To see whether or not pressure fluctuations at $\phi=0.43$ have this feature of fractional Brownian motion, a log-log plot of the prediction error $E_{p}(T) / E_{p}(1)$ against $T$ is shown in Fig. 8. Here, $E_{p}(T)=\frac{1}{\sigma_{e}} \sqrt{\frac{1}{N_{p}} \sum_{i=1}^{N_{p}}\left(p^{\prime}\left(t_{i}+T \Delta t\right)-\hat{p}^{\prime}\left(t_{i}+T \Delta t\right)\right)^{2}}, \quad$ where $\sigma_{e}$ is the standard deviation of the measured pressure fluctua-

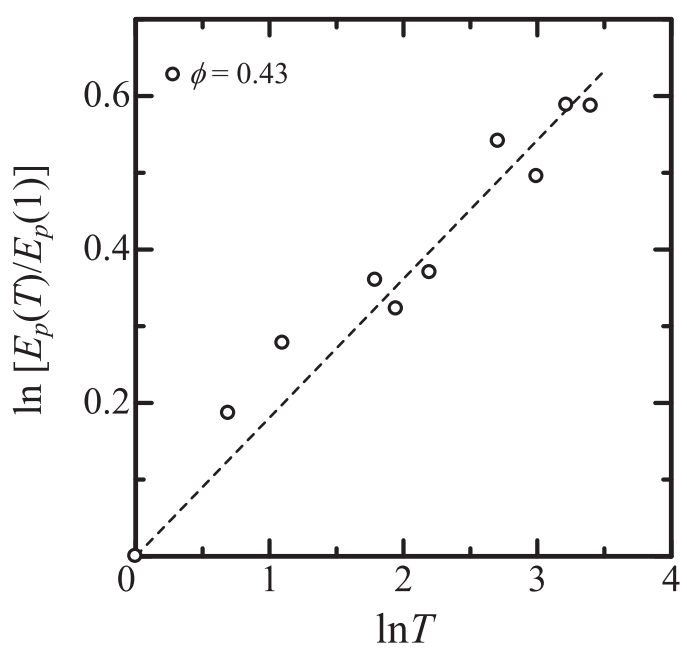

FIG. 8. Log-log plot of prediction error $E_{p}(T) / E_{p}(1)$ as a function of prediction step $T$ at equivalence ratio $\phi=0.43$. The experimental conditions of preheated inlet air flow are the same as those in Fig. 3. The gradient of linear correlation between the $\ln \left[E_{p}(T) / E_{p}(1)\right]$ and $\ln T$ plots, which is the Hurst exponent, is 0.18 , indicating fractional Brownian motion dominated by an antipersistent process. tions and $N_{p}$ is the number of predicted points of pressure fluctuations obtained using the GRBF neural network. As shown in Fig. 8, the $\ln \left[E_{p}(T) / E_{p}(1)\right]-\ln T$ plot exhibits a linear correlation although the data appears to be scattered. The scaling exponent is inferred to be $H=0.18$. $H$ ranges from 0 to 0.5 for positively correlated time series data, i.e., an antipersistent process, while it ranges from 0.5 to 1.0 for negatively correlated data, i.e., a persistent process. This result shows that the dynamic behavior of combustion instability near lean blowout $(\phi=0.43)$ exhibits fractional Brownian motion dominated by an antipersistent process. The permutation entropy of fractional Brownian motion has recently been investigated by Zunino et al. ${ }^{56}$ They showed that permutation entropy gradually decreases with increasing Hurst exponent. According to the theoretical curve of permutation entropy at an embedding dimension of $D=3$ in terms of the Hurst exponent, the permutation entropy $\bar{h}$ is approximately 0.99 at $H=0.2{ }^{55}$ In our preliminary test, the value of $\bar{h}$ estimated for $\phi=0.43$ is approximately 0.98 at $D=3$, which corresponds to that obtained by Zunino et al. ${ }^{56}$ This means that permutation entropy is an important physical quantity for capturing the existence of fractional Brownian motion dominated by an antipersistent process near lean blowout as well as nonlinear forecasting based on a neural network. On the basis of the results obtained by permutation entropy, multifractal analysis, and nonlinear forecasting based on a neural network, it is concluded that the dynamic behavior of combustion instability near lean blowout exhibits a self-affine structure, indicating fractional Brownian motion dominated by an antipersistent process. To the best of our knowledge, this finding has not been discussed in previous studies of combustion instability in a lean premixed gas-turbine model combustor. The characteristics of the combustion dynamics in ducted premixed combustors have recently been quantified by Kabiraj and co-workers ${ }^{11,12}$ and Noble et al. ${ }^{13}$ from the viewpoint of nonlinear dynamics, which is evidence of the importance of nonlinear time series analysis in the treatment of combustion instability. In these studies, ${ }^{1-13}$ the GP method without the surrogate data method was used to reveal the nonlinear characteristics of complex dynamic behavior. The fractional Brownian motion stemming from the stochastic process appears to behave chaotically, but the GP method cannot distinguish the difference between fractional Brownian motion and chaos even if a sufficiently large amount of time series data is available. ${ }^{22}$ Although the use of the conventional GP method to quantify fractal structures may still be important for the combustion community, we consider it unsuitable for the treatment of combustion instability near lean blowout. In this sense, the nonlinear time series analysis we applied in this work, including the translation error, ${ }^{26}$ is progressive and valid for quantifying the nonlinear nature of combustion instability in a lean premixed gas-turbine model combustor.

\section{CONCLUSIONS}

The complexities in combustion instability in a lean premixed gas-turbine model combustor have been characterized from the viewpoint of nonlinear dynamics, focusing on 
characterization of the dynamic behavior of combustion instability close to lean blowout. The dynamic behavior of combustion instability near lean blowout exhibits a selfaffine structure, indicating fractional Brownian motion. It significantly changes to chaotic oscillation when combustion oscillations with slow amplitude modulation appear with increasing equivalence ratio. This is clearly demonstrated by nonlinear time series analysis involving permutation entropy in combination with a surrogate data method, multifractal analysis, and nonlinear forecasting based on a neural network. The characteristics of complexities in combustion instability near lean blowout, revealed using nonlinear time series analysis, have not been reported in previous research on combustion instability in gas-turbine combustors. The nonlinear time series analysis we applied in this work and our previous work ${ }^{26}$ was shown to be valid for the treatment of combustion instability in a lean premixed gas-turbine model combustor.

$\mathrm{OH}^{*}$ and $\mathrm{CH}^{*}$ chemiluminescence emission intensity, which is indicative of fluctuations in the heat release rate in an unstable combustion mode, is also an important physical quantity when considering the dynamic behavior of combustion instability, as has been shown by Kabiraj et al., ${ }^{11}$ who investigated the characteristics of the Poincare section of an attractor constructed from the temporal behavior of the $\mathrm{CH}^{*}$ chemiluminescence emission intensity of thermoacoustic combustion oscillations. The geometrical structure and orbital instability of the attractor constructed from the time series of $\mathrm{OH}^{*}$ and $\mathrm{CH}^{*}$ chemiluminescence emission intensity remain to be elucidated; thus, we will investigate them in terms of the permutation entropy, multifractal structure, and nonlinear forecasting in a future study.

\section{ACKNOWLEDGMENTS}

One of the authors (H.G.) was partially supported by a "Research Grant from Paloma Environmental Technology Foundation" and "Grant-in-Aid for Young Scientists (A) from the Ministry of Education, Culture, Sports, Science and Technology of Japan (MEXT)." The authors would also like to thank Professor Isao Tokuda for the useful comments regarding nonlinear time series analysis and the reviewer of our previous work ${ }^{26}$ for the suggestion of introducing nonlinear time series analysis involving the permutation entropy and nonlinear forecasting based on a neural network.

${ }^{1}$ Y. Huang and V. Yang, Prog. Energy Combust. Sci. 35, 293 (2009).

${ }^{2}$ S. Candel, Proc. Combust Inst. 29, 1 (2002).

${ }^{3}$ T. Poinsot, A. Trouve, D. Veynante, S. Candel, and E. Esposito, J. Fluid Mech. 177, 265 (1987).

${ }^{4}$ K. C. Schadow and E. J. Gutmark, Prog. Energy Combust. Sci. 18, 117 (1992).

${ }^{5}$ W. P. Shih, J. G. Lee, and D. A. Santavicca, Proc. Combust. Inst. 26, 2771 (1996).

${ }^{6}$ T. C. Lieuwen, J. Propul. Power 18, 61 (2002).

${ }^{7}$ A. P. Dowling and A. S. Morgans, Annu. Rev. Fluid Mech. 37, 151 (2005).

${ }^{8}$ S. Tachibana, L. Zimmer, Y. Kurosawa, and K. Suzuki, Nagare 25, 219 (2006).

${ }^{9}$ S. Tachibana, Y. Kurosawa, and K. Suzuki, Proc. Combust. Inst. 31, 3225 (2007).

${ }^{10}$ C. S. Daw, J. F. Thomas, G. A. Richards, and L. L. Narayanaswami, Chaos 5, 662 (1995).
${ }^{11}$ L. Kabiraj, R. I. Sujith, and P. Wahi, J. Eng. Gas Turbines Power 134, 031502 (2012).

${ }^{12}$ L. Kabiraj, A. Saurabh, P. Wahi, and R. I. Sujith, Chaos 22, 023129 (2012).

${ }^{13}$ A. C. Noble, G. B. King, N. M. Laurendeau, J. R. Gord, S. Roy, Combust. Sci. Technol. 184, 293 (2012).

${ }^{14}$ T. Kaminski, M. Wendeker, K. Urbanowicz, and G. Litak, Chaos 14, 461 (2004).

${ }^{15}$ A. K. Sen, G. Litak, T. Kaminski, and M. Wendeker, Chaos 18, 033115 (2008).

${ }^{16}$ H. Gotoda, T. Miyano, and I. G. Shepherd, Phys. Rev. E 81, 026211 (2010).

${ }^{17}$ S. Datta, S. Mondal, A. Mukhopadhyay, D. Sanyal, and S. Sen, Combust. Theory Modell. 13, 17 (2009).

${ }^{18} \mathrm{H}$. Ng, A. Higgins, C. Kiyanda, M. Radulescu, J. Lee, K. Bates, and N. Nikiforakis, Combust. Theory Modell. 9, 159 (2005).

${ }^{19}$ H. A. Abderrahmane, F. Paquet, and H. D. Ng, Combust. Theory Modell. 15, 205 (2011).

${ }^{20}$ P. L. Curto-Risso, A. Medina, A. Calvo Hernarndez, L. Guzman-Vargas, and F. Angulo-Brown, Physica A 389, 5662 (2010).

${ }^{21}$ P. Grassberger and I. Procaccia, Phys. Rev. Lett. 50, 346 (1983).

${ }^{22}$ A. R. Osborne and A. Provenzale, Physica D 35, 357 (1989).

${ }^{23}$ C. Diks, Phys. Rev. E 53, R4263 (1996).

${ }^{24}$ D. Yu, M. Small, R. G. Harrison, and C. Diks, Phys. Rev. E 61, 3750 (2000).

${ }^{25}$ M. Small, D. Yu, and R. G. Harrison, Phys. Rev. Lett. 87, 18101 (2001).

${ }^{26}$ H. Gotoda, H. Nikimoto, T. Miyano, and S. Tachibana, Chaos 21, 013124 (2011).

${ }^{27}$ C. Bandt and B. Pompe, Phys. Rev. Lett. 88, 174102 (2002).

${ }^{28} \mathrm{H}$. Gotoda, K. Michigami, K. Ikeda, and T. Miyano, Combust. Theory Modell. 14, 479 (2010).

${ }^{29}$ G. A. Gottwald and I. Melbourne, Proc. R. Soc. London, Ser. A 460, 603 (2003).

${ }^{30}$ J. Theiler, S. Eubank, A. Lonting, B. Galdrikian, and J. D. Farmer, Physica D 58, 77 (1992).

${ }^{31}$ J. Theiler, Phys. Lett. A 196, 335 (1995).

${ }^{32}$ M. Small and K. Judd, Int. J. Bifurcation Chaos Appl. Sci. Eng. 8, 1231 (1998).

${ }^{33}$ M. Casdaghi, Physica D 35, 335 (1989).

${ }^{34}$ G. Sugihara and R. M. May, Nature 344, 734 (1990).

${ }^{35}$ D. J. Wales, Nature 350, 485 (1991).

${ }^{36}$ A. A. Tsonis and J. B. Elsner, Nature 358, 217 (1992).

${ }^{37}$ J. H. Lefebre, D. A. Goodings, M. V. Kamath, and E. L. Fallen, Chaos 3, 267 (1993).

${ }^{38}$ T. Miyano, H. Morita, A. Shintani, T. Kanda, and M. Hourai, J. Appl. Phys. 76, 2681 (1994).

${ }^{39}$ F. Girosi, M. Jones, and T. Poggio, Neural Comput. 7, 219 (1995).

${ }^{40}$ A. Tikhonov and V. Y. Arsenin, Solutions of Ill-Posed Problem (W. H. Winston, Washington, D.C., 1977).

${ }^{41}$ H. Kantz and T. Schreiber, Nonlinear Time Series Analysis (Cambridge University Press, 1997).

${ }^{42}$ T. Ikeguchi and K. Aihara, Phys. Rev. E 55, 2530 (1997).

${ }^{43}$ R. B. Govindan, K. Narayanan, and M. S. Gopinathan, Chaos 8, 495 (1998).

${ }^{44}$ M. Small and K. Judd, Physica D 117, 283 (1998).

${ }^{45}$ G. Xiaofeng and C. H. Lai, Phys. Rev. E 60, 5463 (1999).

${ }^{46}$ T. Miyano, S. Kimoto, H. Shibuta, K. Nakashima, Y. Ikenaga, and K. Aihara, Physica D 135, 305 (2000).

${ }^{47}$ Y. Xiao, Y. Huang, M. Li, R. Xu, and S. Xiao, Phys. Rev. E 68, 061913 (2003).

${ }^{48}$ D. Haraki, T. Suzuki, H. Hashiguchi, and T. Ikeguchi, Phys. Rev. E 75, 056212 (2007).

${ }^{49}$ T. Suzuki, T. Ikeguchi, and M. Suzuki, Phys. Rev. E 76, 046202 (2007).

${ }^{50}$ M. P. Hanias, I. L. Giannis, and G. S. Tombras, Chaos 20, 013105 (2010).

${ }^{51}$ F. Takens, Lecture Notes in Mathematics (Springer-Verlag, 1981), Vol. 898.

${ }^{52}$ K. P. Harikrishnan, R. Misra, G. Ambika, and R. E. Amritkar, Chaos 19, 043129 (2009).

${ }^{53}$ T. Miyano, T. Moriya, H. Nagaike, N. Ikeuchi, and T. Matsumoto, J. Phys. D 41, 035209 (2008).

${ }^{54}$ H. Gotoda and T. Ueda, Proc. Combust. Inst. 29, 1503 (2002).

${ }^{55}$ H. Gotoda, Y. Asano, K. H. Chuah, G. Kushida, and T. Miyano, Int. J. Heat Mass Transfer 54, 5423 (2009).

${ }^{56}$ L. Zunino, D. G. Perez, M. T. Martin, M. Garavaglia, A. Plastino, and O. A. Rosso, Physics Lett. A 372, 4768 (2008). 